\title{
Evaluation and applications of radiotelemetry in small laboratory animals ${ }^{1}$
}

\author{
Klaas Kramer ${ }^{1}$ and Lewis B. Kinter ${ }^{2}$ \\ ${ }^{1}$ Department of Safety and Environmental Affairs, Free University, DVM, 1081 BT Amsterdam, \\ The Netherlands; and ${ }^{2}$ Safety Assessment, AstraZeneca Pharmaceuticals, Wilmington, Delaware, 19850
}

Submitted 20 November 2002; accepted in final form 12 February 2003

Kramer, Klaas, and Lewis B. Kinter. Evaluation and applications of radiotelemetry in small laboratory animals. Physiol Genomics 13: 197-205, 2003; 10.1152/physiolgenomics.00164.2002.-Radiotelemetry is the "state of the art" for monitoring physiological functions in awake and freely moving laboratory animals, while minimizing stress artifacts. For researchers, especially those in the fields of pharmacology and toxicology, the technique provides a valuable tool for defining the physiological and pathophysiological consequences derived from advances molecular, cellular, and tissue biology and in predicting the effectiveness and safety of new compounds in humans. There is ample evidence that radiotelemetry systems for measuring physiological functions has been sufficiently validated. Today, the technology is an important tool for collection of a growing number of physiological parameters, for contributing to animal welfare (reduction and refinement alternatives), and for reducing overall animal research costs.

rat; mouse; animal welfare; research costs

THE DESIRE TO EXPERIENCE LIFE from the perspective of animals dates from antiquity. Scientists have adapted available technologies to study animals in their quests to unravel and understand biological functions and processes for several centuries; in the most recent of these endeavors, scientists have applied radiotelemetry technology. Radiotelemetry combines miniature sensors and transmitters to detect and broadcast biological signals in animals to remote receiver. The receiver converts the analog frequency signal into a digital signal to be imputed into a computerized data acquisition system. The acquisition system can store, manipulate, format, tabulate, and output the data in accord with the instructions of the user. Currently, radiotelemetry systems can collect blood pressure (BP), heart rate (HR), blood flow (BF), electrocardiogram (ECG) and other biopotentials (EEG, EMG), respiratory rate $(\mathrm{RR}), \mathrm{pH}$, body temperature (BT), and activity indices.

As with all new technology, scientists should be skeptical and demand validations of the new technol-

Article published online before print. See web site for date of publication (http://physiolgenomics.physiology.org).

Address for reprint requests and other correspondence: K. Kramer, Dept. of Safety and Environmental Affairs, Free Univ.-DVM, Van der Boechorststraat 1, 1081 BT Amsterdam, The Netherlands (E-mail: k.kramer@dienst.vu.nl).

${ }^{1}$ This review article was based on work originally presented at the "NHLBI Symposium on Phenotyping: Mouse Cardiovascular Function and Development" held at the Natcher Conference Center, NIH, Bethesda, MD, on October 10-11, 2002. ogy versus current conventional measurement techniques, to the extent possible. However, new technologies make it possible to perform measurements under conditions that have not previously been possible, making direct comparisons with current conventional measurement techniques sometimes impractical.

With the exception of studies of anesthetic agents and certain other types of experiments involving acutely painful/stressful procedures, it is generally acknowledged that the quality of physiological measurements collected from conscious unstressed animals is superior, since they are collected under conditions that best represent the normal state of the animal, are least influenced by chemical, stress, and psychological factors and (where appropriate) are most predictive of the results that would be achieved in human beings $(1,2)$. When monitoring physiological parameters in conscious animals, it is easiest to use noninvasive methods such as surface electrodes for monitoring an ECG or a tail-cuff manometry for monitoring BP. However, use of noninvasive techniques can introduce significant artifacts, as these techniques often require physical restraints and high levels of technician interaction to ensure that surface connections stay in place and do not accidentally damage, or be damaged by, the animal. The literature extensively documents the effects of restraint on laboratory animals, including increases in BT, HR, and BP, plasma levels of epinephrine and norepinephrine, changes in responses to pharmaceuticals, and decreased food intake and body weight (49, 51). Temporary use of invasive methods to implant 
sensors under the skin and/or within body cavities can eliminate the experimental artifacts associated with noninvasive procedures. Development of modern microsurgical techniques and surgical recovery procedures has greatly facilitated the use of indwelling catheters (for monitoring fluid pressure/removing fluid samples), electrodes (for monitoring biopotentials and temperatures), and devices (e.g., flow probes, vascular cuffs) (86).

When using indwelling catheters, sensors, and/or electrodes, several methods (including wireless radiotelemetry) for accessing the information from the sensor and forwarding it to a recording system are available; the advantages and disadvantages of each of these methods are extensively summarized in an earlier review (51). Although wireless radiotelemetry technology for monitoring laboratory animals has existed for at least 50 years $(11,51)$, it has only been in the last 10 years that affordable, reliable, and easy-touse commercial products have been really available for monitoring physiological signals in laboratory animals [Biomedic Data Systems (BMDS), Seaford, DE 19973; Data Sciences International (DSI), St. Paul, MN 55126; Konigsberg Instruments, Pasadena, CA 91107; Mini Mitter, Sunriver, OR 97707; and Star Medical, Arakawa-ku, Tokyo 116, Japan]. Understandably, availability has resulted in a significant increase in the use of implantable radiotelemetry in biomedical research, and several authors have highlighted its advantages: 1) There is a reduction of distress compared with conventional measurement techniques. Telemetry represents the most humane method for monitoring of physiological parameters in conscious, freely moving laboratory animals (11).2) One may eliminate of use of restraints, which can alleviate a potential source of experimental artifact and inter-animal variability (75). 3) There is a reduction of animal use by $60-70 \%$ in single studies (85) and by more than $90 \%$ in multiple studies (43). 4) Refinement in animal use permitted virtually unrestricted continuous data collection (days, weeks, months, or more) without the need of any special animal care (11). 5) Available for use in all laboratory species, from mice to monkeys (49), and most recently fish (Snelderwaard PC, Van Ginneken V, Witte F, Voss HP, and Kramer K, unpublished observations).

As well, authors have highlighted the disadvantages of implantable radiotelemetry in biomedical research: 1) There is a cost to acquire the requisite equipment (usually completely recovered through reduced animal use). 2) Specialized training/certifications are required to surgically prepare and study telemeterized animals. 3) Continuous or regular scheduled sampling generates large amounts of data (e.g., 5-min scheduled sampling over an 8-wk period will generate more than 16,000 data points per parameter), which can lead to analysis problems. 6) Dedicated space must be found within the animal facility in which to conduct studies.

Although the use of telemetry technology is well established in many and diverse areas of animal research, we have limited the scope of this review to recent telemetry applications in small laboratory animals.

\section{APPLICATIONS AND EVALUATION}

Transmitter implantation. Telemetry systems consist of an implantable hermetically sealed sensor/ transmitter (transmitter) unit coated with a biocompatible material and an external receiver system. In some systems the implantable sensor system is separate from an external transmitter system, and nonimplanted oral telemetry "capsules" for use in the gastrointestinal tract are also described (46). Implantation of sensor/transmitters requires facilities for sterile recovery surgical procedures, expertise with microsurgical techniques (86), and is beyond the scope of this review. Using sterile procedures, the technician affixes the transmitter in a convenient subcutaneous pocket or directly within the peritoneal cavity. For ECG and EEG measurements the positive and negative electrodes are placed subcutaneously (47); for BP measurements one or more fluid-filled catheters are placed into the appropriate blood vessel [usually the carotid artery, thoracic aorta, femoral artery, pulmonary artery, or abdominal aorta $(18,62)]$. For measurements of respiratory pressure changes, a fluid-filled catheter is introduced into the pleural cavity (67). Once the equipment is placed and checked for proper function, all incisions are closed and the animals recovered from surgery are permitted 1-2 wk recovery with appropriate analgesics before studies are conducted.

Critics of this technology correctly inquire as to the effects of the weight and volume of the implanted transmitter on animal behavior and stress and on physiological functions (Morton DB, Hawkins P, Bevan R, Heath K, Kirkwood J, Pearce P, Scott E, Whelan G, and Webb A, unpublished observations). Animals seem to tolerate the implantations of the transmitter without obvious problems, so long as high-quality sterile techniques are used $(7,64)$. Baumans et al. (7) assessed changes in body weight and behavior of BALB/c and 129/Sv mice, after implantation of an intra-abdominal transmitter (DSI). For behavior, the automated Laboratory Animal Behaviour Observation Registration and Analysis System (LABORAS; Metris System Engineering, Hoofddorp, The Netherlands) was used. With LABORAS climbing, locomotion, immobility, grooming, eating, and drinking behaviors can be monitored without disturbing the animal $(16,74)$. During the first days after surgery, body weight and climbing, locomotion, and eating were decreased in both strains, whereas grooming and immobility were increased. These changes were more pronounced in the transmitter-implanted animals than in the sham-operated animals, indicating temporarily impaired well being; however, within 2 wk after surgery there were no differences between the two treated groups, and all animals were judged to be fully recovered.

Validation. Validation of a telemetry system includes calibration/verification of the sensor, conformation that the sensor signal is accurately received and 
accumulated within the data acquisition system, conformation that all post-collection data processing programs and subroutines are functioning in accord with specifications, and conformation of the accuracy and precision of the data outputs. When multiple animals/ systems are studies simultaneously, validation must include verification of the accuracy and integrity of each data channel. For the purpose of this review, we will focus on validation of the sensor/transmitter function and refer the reader to manufacturers of telemetry systems for validation of data acquisition systems. Suffice it to say that telemetry systems are easily validated for use within studies conducted under Good Laboratory Practice (Kinter LB, personal experience).

It is necessary to validate telemetry sensor/transmitter signals for accuracy over the anticipated physiological range and for stability over the duration of intended use. Experimental approaches to validate transmitters include simultaneous measurements in single or in separate groups of animals, comparing the telemetry signal to that of one or more conventional technique(s). When comparing telemetry and conventional techniques, small differences in absolute values can be anticipated based upon differential placement of catheters and electrodes. Short-acting pharmacological agents and manipulations can be used to induce acute changes in physiological parameters to test dynamic ranges; alternatively, circadian variations can be used. Stability is best approached through measurements collected after implantation and again before the transmitter is removed (but before the power source fails). The results of a validation protocol should demonstrate that the data collected using the telemetry and conventional technologies "mirror" each other in both relative (increase/decrease with time) and absolute values; differences in absolute values can be post-corrected using with knowledge of the offset value, if necessary.

The most straightforward approach to validation of the transmitter is during the surgical implantation procedure: Step 1) Conduct the procedures recommended by the manufacturer to verify the specific device to be implanted and any factory-established offset parameters. Step 2) Implant the transmitter using sterile procedures and in accord with a protocol approved by an appropriate ethical review committee. Step 3) Calibrate and connect the conventional technology to which the telemetry signal will be compared. This may require acute catheterization of another blood vessel, parallel placement of biopotential leads, etc. Step 4) Simultaneously collect data using the two technologies and compare the outputs; $4 a$ ) if the outputs are within previously established acceptable limits (e.g., $\pm 10 \%$ ), continue; $4 b$ ) if not, diagnose the difference, correct, and repeat. Step 5) Administer appropriate doses (usually intravenously) of short-acting pharmacological agents or manipulations to induce excursions in primary parameters; simultaneously record results and compare outputs (as above). Note that these agents must "clinical grade" in terms of purity, formulation, and sterility; clinical supplies are recommended (see Ref. 45 for agents and doses). 5a)
$\mathrm{BP} / \mathrm{HR}$ (isoproterenol, epinephrine, norepinephrine); 5b) ECG [calcium channel antagonist (P-R interval), class III antiarrhythmic (Q-T interval)]; 5c) respiratory rate (hypercarbia to stimulate; volatile anesthetic to depress); and 5d) BT (usually a multi-point with a rectal temperature). Step 6) Remove the conventional technology, close the remaining incisions, and recover the animal from anesthesia. Step 7) To determine longterm stability of the signal, reanesthetize the telemeterized animal prior to transmitter failure and repeat step 4 (and possibly step 5). The record differences. Repeat step 6 or euthanize the preparation and recover implanted devices for refurbishing.

The following include examples of validation of "telemeterized" physiological functions in laboratory species.

Blood pressure, electrocardiogram, and heart rate. BP, ECG, and HR, as well as BT sensors, measured with radiotelemetry in nonrodent species (dog, minipigs, monkeys) can be validated using the general procedure described above. Use of sensors for these parameters in rodents has been extensively validated and documented in recent literature $(13,14,23,49,51)$. In addition, Mills et al. (62) described, for the first time, the possibility to record systolic, diastolic, and mean $\mathrm{BP}$, as well as HR and locomotor activity, in freely moving mice, living in their home cage, for several months. In general, it is not feasible to conduct simultaneous measurements using telemetry and conventional technologies during transmitter implantation in rodents, due to the small size of their blood vessels, so parallel study groups are recommended. In these species, blood pressure catheters are usually inserted into the abdominal aorta just caudal to the left renal artery (or via the carotid artery), while the body of the transmitter is positioned in the peritoneal cavity. Because the device was developed for abdominal aorta implantation, its use has not been feasible in studies where peritoneal volume is critical, i.e., in pregnant mice. Butz and Davisson (18) developed an alternative approach for measuring $\mathrm{BP}$ in mice, whereby thoracic aorta implantation of the pressure-sensing catheter is combined with subcutaneous placement of the transmitter body along the right flank. Butz and Davisson (18) used for this study female C57BL/6 or BPH/5 mice, a strain derived from the cross of inbred hypertensive and hypotensive mouse strains. The results of their study showed that the above-mentioned approach is a reliable method with which to measure, under stressfree conditions, mean arterial pressure and HR recordings for 50-60 days in mice weighing $22 \mathrm{~g}$ on average but as small as $17 \mathrm{~g}$, even before, during, and after pregnancy.

However, although placing the BP catheter in the carotid rather than in the abdominal aorta of the mouse can permit a less invasive operation procedure, one must be aware of the fact that the Circle of Willis is not completely developed in some strains of mice, and thus the carotid approach is not advised for these strains $(6,88)$. 
Electroencephalogram. Validation of biopotential signals (EEG, EMG, ECG) is generally qualitative rather than quantitative process, focusing upon obtaining appropriate waveform morphology and minimizing artifacts. An exception is where specific software is used to "strip" biopotential waveform data into tabular formats (e.g., ECG peak heights and peak intervals); in these cases it is necessary to establish the sensitivity of the telemetry system to detect specific changes in waveform morphology. Species differences must also be considered [e.g., optimal ECG lead placements and differences in cardiac depolarization vector (47)]. Livezey and Sparber (59) were one of the first to describe an implantable radiotelemetry system to measure EEG in rats; a few years later Champeroux et al. (19) described radiotelemetry for EEG monitoring in conscious rabbits. Kant et al. (42) determined the effects of chronic stress on sleep, using a rodent paradigm of around-the-clock signaled intermittent foot shock in which some rats can pull a chain to avoid/ escape shock while another group of rats is yoked to the first group. Sleep was measured by using a telemetry device for recording the EEG. Rats were implanted with four stainless steel electrode screws over the frontal cortex and the cingulate cortex; a bipolar electrode was placed in the dorsal hippocampus. The electrodes were connected to a headset that was cemented to the skull. The transmitter was inserted into a small pocket behind the shoulder blades, and the leads were connected to the headset. The results of this study suggested that the stress and the performance requirements of the paradigm caused sleep disruption. Cotugno et al. (21) recommended the radiotelemetry system of EEG recording for the following reasons: 1) subcutaneous placement of the transmitter; 2) EEG recordings around-the-clock for weeks; 3) surgery is quite simple; and 4) the system is commercially available.

Antier et al. (4) showed the influence of neonatal focal cerebral hypoxia-ischemia (HI) on sleep-walking pattern, on electrocorticogram $(\mathrm{ECoG})$ power spectra, and on locomotor activity (LA) in adult Wistar rats. The animals were implanted with a radiotelemetry implant for simultaneous measurements of ECoG and LA. Two silver custom-made ECoG electrodes were implanted over the frontal cortex: one electrode at the coordinates relative to bregma, and a second electrode over the right parietal cortex. The authors indicated that the animals exposed to a neonatal unilateral cerebral HI present significant ECoG activity, sleep-waking pattern, and behavioral disturbances when adults. Timofeeva and Gordon (83) implanted transmitters in adult female rats and recorded EEG, core temperature, and motor activity before and after exposure to the acetylcholinesterase inhibitor chlorpyrifos (CHP) and muscarinic agonist oxotremorine (OX). Their data showed that activation of muscarinic receptors is responsible for most of the EEG and behavioral alterations induced by CHP. Cortical arousal together with increased quiet waking and decreased states of sleep after CHP occurred independently from inhibition of motor activity and lowering of core temperature. Because the EEG is a sensitive parameter, radiotelemetry provides a means of assessing subtle neurological changes following exposure to neurotoxicants.

Finally, Mumford and Wetherell (66) described a method of measuring EEG in guinea pigs by means of temporarily attached superficial electrodes with radiotelemetry equipment, an alternative approach to more conventional methods of monitoring EEG in animals, which necessitate surgical implantations of electrodes. Superficial electrodes were compared with the cortical screw electrode technique in a nerve agent-induced seizure model. The authors demonstrated the possibility of the collection of meaningful data using temporarily attached superficial electrodes and have shown that, in a nerve agent seizure model, seizure activity in the freely moving nontethered guinea pig may be identified using this equipment. They also have discussed the advantages and disadvantages of the technique with respect to resource conservation and the benefits to animal welfare.

Electromyogram. Kant et al. (42) and Sei and Morita (76) implanted two Teflon-coated stainless steel wire electrodes bilaterally in the nuchal muscle and used radiotelemetry to measure EMG in freely moving rats. Nakajima et al. (68) measured contractile activity of the gastrointestinal (GI) tract in conscious unrestrained rats. The transmitter body was fixed in the peritoneal cavity of the rat, and the sensing catheter was sutured into the serosa in the gastric antrum. Gastric motility of freely moving rats could be continuously recorded for up to 60 days. Recently, Meile and Zittel (61) published telemetric transmission of rat gastrointestinal EMG signals that were comparable to those given in the literature. Two $\mathrm{NiCr}$ electrodes were sutured to the jejunum and connected to an implantable EMG transmitter; EMG signals were transmitted to a receiver placed at the bottom of the rat's home cages. Langenbach et al. (55) used a radiotelemetry system to chronically record muscle activity in rabbits. The results of this study suggest that the tested device provides a good measure of the muscle activity pattern at the sampled region with a minimum of signal dropouts. The device is small enough to be implanted in rats, and the telemetry technique enables the recording of daily muscle activity in freely moving animals without interference of the observer.

Respiratory rate. Respiratory rate $(\mathrm{RR})$ can be derived from the pleural waveform using an intrapleural catheter (67). A new method for measuring $R R$ in rats (12) and mice (Brockway RV and Huetteman DA, 2001, personal communication,) has been developed, in which the RR signal is derived from the arterial pressure waveform, without the need of an extra intrapleural catheter, and has the potential to provide unique data in long-term cardiovascular studies (48). Although the influence of respiration on $\mathrm{BP}$ waveform is a well-know physiological phenomenon, the RespiRATE software (Data Sciences International) is the first commercial product to employ this effect to derive respiratory information from a BP signal. The pleural 
pressure waveform is derived by fitting a curve to beat-to-beat systolic pressures, from which $R R$ is derived (48).

Telemetry applications in inbred rodent strains and transgenic models for cardiovascular disease. During the last decade there has been a large increase in using transgenic models, and specifically mouse models, to investigate the causes of, and potential treatments for, cardiovascular diseases $(9,26,38,73)$. Expression of abnormal quantities or altered forms of gene products in an intact animal provides a powerful tool by which to gain insight into the role of expression of specific genes in cardiac growth, development, and function (63). Examples of transgenic mouse models relevant to cardiovascular physiology and diseases are extensively described and discussed in recent reviews (20, 24, 28, 38). Miniaturized cardiovascular telemetry technologies suitable for mice greatly expand the quality and quantity of physiological data to be extracted from murine transgenic models. Recent measurements of ECG, HR, and BP using telemetry in transgenic mouse models have resulted in qualitative and quantitative changes in definitions of ECG waveforms, in baseline values of $\mathrm{HR}$ and $\mathrm{BP}$, and in responses to autonomic agents, compared with nontransgenic control mice $(22,26$, $31-33,36,37,39-41,60,69,79,81,89)$. For example, Gehrmann et al. (31) developed HR variability (HRV) analysis in the mouse for characterization of $\mathrm{HR}$ dynamics, modulated by vagal and sympathetic activity, and concluded that phenotypic screening for HR regulation in mice further enhanced the value of the mouse as a model of inheritable electrophysiological human disease. In transgenic mice with a specific eightfold atrial overexpression of human $\beta_{1}$-adrenoceptor $\left(\beta_{1^{-}}\right.$ AR) (60) or with a cardiac-specific overexpression of the $\alpha$-subunit of the GTP-binding protein $\left(\mathrm{G}_{\mathrm{s}} \alpha\right)$ (84), no effect of $\left(\beta_{1}-\mathrm{AR}\right)$ overexpression was found in the HR, whereas HRV in transgenic mice was decreased, and $\mathrm{G}_{\mathrm{s}} \alpha$ overexpression significantly increased HR compared with the control mice, whereas HRV was again decreased. In another study of two commonly used inbred mouse strains [A/J and C57BL/6J (B6)], telemetry showed that BT was generally higher and HR significantly lower in $\mathrm{B} 6$ than $\mathrm{A} / \mathrm{J}$ mice, underscoring strain differences in baseline physiological values (36). Johansson et al. (39) recorded HR, ECG, and BT in mice lacking thyroid hormone receptor- $\beta$ (TR- $\beta$ ) or TR- $\alpha 1$ and $-\beta($ TR- $\alpha 1 / \beta)$ and found that the TR- $\alpha 1 / \beta-$ deficient mice had a reduced HR compared with wildtype controls, and the TR- $\beta$-deficient mice showed an elevated HR. ECG revealed that the TR- $\beta$-deficient mice had a shortened Q-T interval in contrast to the TR- $\alpha 1 / \beta$-deficient mice, which exhibited prolonged P-Q and Q-T intervals. Heterozygous knockout (KO) mice with half the wild-type expression of the vesicular monoamine transporter (VMAT2) can suddenly die in midlife; Itokawa et al. (37) examined ECG data telemetered from freely moving heterozygote and wildtype littermate mice. And found many ECG parameters were indistinguishable in these two strains. However, heterozygous mice displayed prolonged Q-T intervals, suggesting vulnerability to lethal ventricular arrhythmias in these animals and the existence of a candidate gene for interindividual differences in vulnerability. Shusterman et al. (79) measured HRV and pharmacological responses in three different experimental settings: 1) wild-type FVB mice, a common strain used in transgenic overexpressors; 2) C57BL6/ SV129 mice, the most common strain found in genetargeted mice; and 3) TNF- $\alpha$ mice (on a FVB background) that develop a dilated cardiomyopathy by 12 wk of age. They concluded that interstrain differences in autonomic nervous system activity are important when studying transgenic models; that the homeostatic states of autonomic nervous system activity are strain specific, leading to differences in HR, responses to pharmacological agents, and pathophysiological changes in disease models; and that strain-specific differences should be considered in selecting the strains of mice used for transgenic and gene targeting experiments.

Stauss et al. (81) compared BP variability of eNOS KO mice (lacking endothelial nitric oxide synthase, eNOS) with their respective wild-type controls. One day after carotid artery cannulation, BP was recorded in these conscious mice. During resting conditions, BP variability was markedly enhanced in $\mathrm{KO}$ mice compared with wild-type mice. In another paper, baseline $\mathrm{BP}$ values were higher in AT2 receptor KO than in wild-type mice (32). Davisson et al. (22) reported an inbred mouse strain (BPH/5) with mildly elevated BP which spontaneously develops a syndrome that bears close resemblance to preeclampsia in humans and concluded, given that preeclampsia likely has an important genetic component, this inbred genetic strain may provide an opportunity for studying its genetic pathophysiology. Radiotelemetry was also used in combination with a treadmill test in transgenic mice overexpressing ventricular myosin regulatory light chain; the capacity for exercise at higher belt speed $(27 \mathrm{~m} / \mathrm{min})$ was significantly decreased in transgenic mice compared with nontransgenic mice (26).

Finally, circadian BP and HR variations were measured by radiotelemetry in freely moving normotensive and hypertensive rats $(3,57,77,91)$.

Telemetry and laboratory animal welfare. In their book Stress and Animal Welfare, Broom and Johnson (15) have defined welfare as "the state of an individual as regards its attempts to cope with its environment", thus the ability of an animal to cope with or adapt to internal and external stressors. To investigate animal welfare, the impact of stress on physiological parameters, such as body weight, hormonal levels in plasma and/or urine, HR, BP, and BT can be measured (15, 25, $34,49,56,71,87)$. When measurements of the physiological parameters are performed via conventional measurement techniques, the results must be interpreted with caution, since these conventional techniques have by themselves contribute to animal stress.

Well-known examples include the decrease in body weight (82), the increase in catecholamine levels (53), and increased BP (54) when rats are immobilized. 
Intra-arterial catheterization of rats has been shown to cause decreased food intake as well as body weight loss (70). BP measurements in mice via the tail-cuff technique caused increases in both HR and BP (52). Recently, Duke et al. (25) described the effects of routine cage changing on cardiovascular and behavioral parameters in male Sprague-Dawley rats and concluded that common animal husbandry procedures, such as moving rats to a clean cage, can induce transient, but significant, cardiovascular and behavioral changes. Since radiotelemetry with an implantable transmitter provides a way to obtain accurate and reliable physiological measurements from awake and freely moving animals in their own environment (11), it is a valuable tool to investigate animal welfare without the overlay of stress-producing factors associated with conventional techniques $(17,30,49,51)$.

The humane use of laboratory animals requires implementation of the "three Rs" (replacement, reduction, and refinement) of Russell and Burch (72). The "replacement" alternative is defined by Russell and Burch as any scientific method employing nonsentient material, which may replace methods that use conscious vertebrates. The "reduction" alternative directs the use of experimental designs and statistical methods to support the minimum number of animals required to test the hypotheses at an appropriate power. Finally, the "refinement" alternative focuses upon methods to minimize or eliminate pain and distress and to enhance laboratory animal well-being. Radiotelemetry technology can contribute directly to "reduction" and "refinement" alternatives.

Telemetry and reduction alternatives. Radiotelemetry can substantially reduce animal use and associated research costs by increasing the number of parameters that can be collected from a group of animals and thereby eliminating the need for satellite groups or separate studies to collect the same data, by permitting the use of blocked study designs, and by increasing the data precision in both individuals and study groups. Combination of cardiovascular and general toxicity endpoints in single-dose and dose-ranging toxicity studies can satisfy both general toxicity and safety pharmacology testing requirements for pharmaceuticals in a single study (see ICH S7A; Ref. 1). Changes in $\mathrm{BP}, \mathrm{HR}$, and ECG parameters detected by radiotelemetry may provide direct evidence of a dose-limiting effect $(44,65)$, eliminating the need for more animals to evaluate higher doses for toxicity.

An attribute of telemeterized preparations is that, with suitable precautions, animals can be reused to study different doses and test regimens (blocked study designs), to verify individual efficacy and toxicity responses, and even separate studies. Use of blocked study designs filters out the inter-animal variation and reduces the numbers of animals needed to obtain the same level of statistical power for evaluating dose/ treatment responses $(27,90)$. Because radiotelemetry permits continuous or periodic collection of cardiovascular data over prolonged periods of time, it is compatible with the use of randomized block designs, replac- ing conventional, completely randomized, study designs. The reduction in animal use achieved by using randomized block designs instead of completely randomized designs is $75 \%$, without loss of statistical power (45). A variant of the randomized block design is one in which all animals receive all treatments, but in ascending (vehicle, then low, mid, and high dose) or descending (high, then mid, low, and vehicle) order. This design has the same improved statistical power as the randomized block design but may require an additional time control to separate treatment effects from time effects (45).

Because of the growing lifetime of radiotelemetry implants (up to a year and longer), investigators may consider reusing experimental animals to study additional doses, different treatments, alternate routes of administration, or to verify response differences in individual animals. The total reduction in animal use achieved by eliminating a study, by using randomized blocking, and by reusing animals in multiple studies is $>90 \%$ (45). Although it is beyond dispute that reuse can substantially reduce research costs and overall animal use, reusing instrumented animals for additional procedures is controversial and subject to specific regulations, in part because previous technologies (arterial punctures, externalized catheters and electrodes, etc.) contributed cumulatively to animal discomfort/stress. Because miniaturized implanted telemetry systems obviate those concerns, the authors propose that it is now appropriate to readdress regulatory aspects of reuse of telemeterized preparations in light of the contributions these preparations can make to science and the "reduction" aspect of Russell and Burch, without compromising animal welfare.

Studies in our own laboratory have shown that a reduction in animal use up to $70 \%$ can be achieved when the data obtained in our mice cardiotoxicity studies are compared with literature data obtained via morphometry or histology studies (85). Fraser et al. (29) compared a telemetric BP monitoring system with a tail-cuff BP measuring system in both adrenocorticotropic hormone (ACTH)-treated and sham-treated rats and concluded that the results measured with telemetry in this study were very similar to the results measured with the tail-cuff method and that telemetry allows for a longer period of measurement, giving greater power to the study so that fewer animals are needed.

Telemetry and refinement alternatives. Radiotelemetry also offers refinement alternatives by reducing animal stress and discomfort, improving the quality and precision of experimental data, and reduces costs. In freely moving mice and rats equipped with cardiovascular radiotelemetry transmitters and monitored in their home cages, HR and BP values were found to be much lower compared with published values $(8,10,47$, $49,50,77)$. As animals implanted with a radiotelemetry transmitter are best monitored while in their home cages, no additional dedicated laboratory space other than that used to house the animals is necessary. No isolation from other animals of the same species is 
required, and no continuous monitoring by trained laboratory staff is required during the conduct of a study, as is required for almost all other cardiovascular preparations.

Since radiotelemetry permits investigation of drug effects in freely moving, unrestrained animals, it facilitates animal models that mimic the clinical conditions of drug investigation, considered as a contribution to the concept of refinement (58).

\section{CONCLUSIONS}

Implantable radiotelemetry is now a viable and commercially available alternative to conventional methods for measurements of physiological functions in biomedical research. There are currently more than 300 peer-reviewed publications (source: Data Sciences International) that include functions measured via fully implanted telemetry in laboratory species from mice to monkeys, including fish (49; and Snelderwaard et al., unpublished observations). Several of these publications describe validation studies $(3,8,10$ 14, 23, 62) concerning the accuracy, utility, or safety of the devices. The scientific literature provides several compelling arguments for the use of implantable radiotelemetry as a more appropriate alternative to traditional methods of monitoring laboratory animals in terms of quality and quantity and the data collected, animal welfare (reduction and refinement alternatives), and to reduce overall animal research costs. Whereas the use of implanted telemetry in rodents may once have been viewed as a technical "tour-de-force" but with significant cost and dubious value, the advent of transgenic rodent technologies and the time and expense to produce transgenic models has made rodent telemetry a cost-effective necessity for the modern research laboratory.

Just as other technology-based products, continuing progress in electronics, battery, sensor, and material technologies will bring further advances in implantable radiotelemetry. The most significant innovations will include further miniaturization, expansion of the numbers of parameters that can be monitored simultaneously, and introduction of new sensors for regional blood flows, concentrations of inorganic and organic substances [e.g., an amperometric glucose biosensor implanted subcutaneously in dogs $(5,78)]$, and radioisotopes in blood and other body compartments.

\section{REFERENCES}

1. Anonymous. Guidance for Industry. S7A Safety Pharmacology Studies for Human Pharmaceuticals. US Department of Health and Human Services, Food and Drug Administration, Center for Drug Evaluation and Research, Center for Biologics Evaluation and Research, ICH, July, 2001 (http://www.ifpma.org/ich5s. html).

2. Anonymous. ICH Guideline on Safety Pharmacology Studies for Assessing the Potential for Delayed Ventricular Repolarization (QT Interval Prolongation) by Human Pharmaceuticals (S7B). Step 2, 2002 (http://www.ifpma.org/ich5s.html).

3. Anderson NH, Devlin AM, Graham D, Morton JJ, Hamilton CA, Reid JL, Schork NJ, and Dominiczak AF. Telemetry for cardiovascular monitoring in a pharmacological study.
New approaches to data analysis. Hypertension 33: 248-255, 1999.

4. Antier D, Zhang BL, Mailliet F, Akoka S, Pourcelot L, and Sannajust F. Effects of neonatal focal cerebral hypoxia-ischemia on sleepwalking pattern, ECoG power spectra and locomotor activity in the adult rat. Brain Res 807: 29-37, 1998.

5. Atannasov P, Yang S, Salehi C, Ghindilis AL, Wilkins E, and Schade D. Implantation of a refillable glucose monitoringtelemetry device. Biosens Bioelectron 12: 669-680, 1990.

6. Barone FC, Knudsen DJ, Nelson AH, Feuerstein GZ, and Willette RN. Mouse strain differences in susceptibility to cerebral ischemia are related to cerebral vascular anatomy. $J$ Cereb Blood Flow Metab 13: 683-692, 1993.

7. Baumans V, Bouwknecht JA, Boere H, Kramer K, Van Lith HA, Van de Weerd HA, and Van Herck H. Intra-abdominal transmitter implantation in mice: Effects on behaviour and body weight. Anim Welfare 10: 291-302, 2001.

8. Bazil MK, Krulan C, and Webb RL. Telemetric monitoring of cardiovascular parameters in conscious spontaneously hypertensive rats. J Cardiovasc Pharmacol 22: 897-905, 1993.

9. Berul CI, Aronnovitz MJ, Wang PJ, and Mendelsohn ME. In vivo cardiac electrophysiology studies in the mouse. Circulation 94: 2641-2648, 1996.

10. Brockway BP, Mills PA, and Azar SH. A new method for continuous chronic measurement and recording of blood pressure, heart rate, and activity in the rat via radio-telemetry. Clin Exp Hypertens 3: 885-895, 1991.

11. Brockway BP and Hassler CR. Application of radio-telemetry to cardiovascular measurements in pharmacology and toxicology. In: New Technologies and Concepts for Reducing Drug Toxicity. Boca Raton: CRC, 1993, p. 109-132.

12. Brockway RV and Huetteman DA. A new method for chronic measurement of respiratory rate in conscious freely moving rats (Abstract). 50th AALAS National Meeting Indianapolis 1998, p. 28.

13. Brockway BP, Mills P, and Kramer K. Fully implanted radio-telemetry for monitoring laboratory animals. Lab Anim 27. 40-46, 1998.

14. Brooks D, Horner RL, Kozar LF, Waddell TK, Render CL, and Phillipson EA. Validation of a telemetry system for longterm measurement of blood pressure. J Appl Physiol 81: 10121018, 1996.

15. Broom DM and Johnson KG. Stress and Animal Welfare. Chapman and Hall, 1993.

16. Bulthuis RJA, Bergman AF, Nijessen S, Schlingmann F, Tolboom J, Remie R, Van de Weerd HA, Van Loo PLP, Baumans V, and Van Zutphen LFM. Automated behaviour classification: the LABORAS project. Harmonisation of Laboratory Animal Husbandry, edited by O'Donoghue PM. Basel: RSM Press, 1996, p. 17-18.

17. Bunag RD. Facts and fallacies about measuring blood pressure in rats. Clin Exp Hypertens 5: 1659-1681, 1983.

18. Butz GM and Davisson RL. Long-term telemetric measurement of cardiovascular parameters in awake mice: a physiological genomics tool. Physiol Genomics 5: 89-97, 2001.

19. Champeroux $\mathbf{P}$, Lala $\mathbf{P}$, and Richard S. Use of Radiotelemetry for Electroencephalogram Monitoring in Conscious Rabbits. Edinburgh: Br Toxicol Soc, 1994.

20. Christensen G, Wang Y, and Chien KR. Physiological assessment of complex cardiac phenotypes in genetically engineered mice. Am J Physiol Heart Circ Physiol 272: H2513-H2524, 1997.

21. Cotugno M, Mandile N, D'Angiolillo D, Montagnese P, and Giuditta A. Implantation of an EEG telemetric transmitter in the rat. Ital J Neurol Sci 17: 131-134, 1996.

22. Davisson RL, Hoffmann DS, Butz GM, Aldape G, Schlager G, Merill DC, Sethi S, Weiss RM, and Bates JN. Discovery of a spontaneous genetic mouse model of preeclampsia. Hypertension 39: 337-342, 2002.

23. Deveney AM, Kjellstrom A, Forsberg T, and Jackson DM. A pharmacological validation of radio-telemetry in conscious freely moving rats. J Pharmacol Toxicol Methods 40: 71-79, 1998. 
24. Doevendans PA, Daemen MJ, De Muinck ED, and Smits JF. Cardiovascular phenotyping in mice. Cardiovasc Res 39: 34-49, 1998.

25. Duke JL, Zammit TG, and Lawson DM. The effects of routine cage changing on cardiovascular and behavioral parameters in male Sprague-Dawley rats. Contemp Top Lab Anim Sci 40: $17-20,2001$.

26. Fewell JG, Osinska H, Klevitsky R, Ng W, Sfyris G, Bahrehmand F, and Robbins JA. A treadmill exercise regimen for identifying cardiovascular phenotypes in transgenic mice. Am $J$ Physiol Heart Circ Physiol 273: H1595-H1605, 1997.

27. Festing MF. The scope for improving the design of laboratory animal experiments. Lab Anim 26: 256-268, 1992.

28. Franz WM, Mueller OJ, Hartong R, Frey N, and Katus HA. Transgenic animal models: new avenues in cardiovascular physiology. J Mol Med 75: 115-129, 1997.

29. Fraser TB, Tutner SW, Mangos GJ, Ludbrook J, and Whitworth JA. Comparison of telemetric and tail-cuff pressure monitoring in adrenocorticotrophic hormone-treated rats. Clin Exp Pharmacol Physiol 28: 831-835, 2001.

30. Gärtner K, Buttner D, Dohler K, Friedel R, Lindena J, and Trautschold I. Stress response of rats to handling and experimental procedures. Lab Anim 14: 267-274, 1980.

31. Gehrmann J, Hammer PE, Maguire CT, Wakimoto H, Triedman JK, and Berul CI. Phenotypic screening for heart rate variability in the mouse. Am J Physiol Heart Circ Physiol 279: H733-H740, 2000.

32. Gross V, Milia AF, Plehm R, Inagami T, and Luft FC. Long-term blood pressure telemetry in AT(2) receptor-disrupted mice. J Hypertens 18: 955-961, 2000.

33. Gross V, Plehm R, Tank J, Jordan J, Diedrich A, Obst M, and Luft FC. Heart rate variability and baroreflex function in AT2 receptor-disrupted mice. Hypertension 40: 207-213, 2002.

34. Harkin A, Connor TJ, O’Donnell JM, and Kelly JP. Physiological and Behavioral responses to stress: what does a rat find stressful? Lab Anim 31: 42-50, 2002.

36. Hoit BD, Kiatchoosakun S, Restivo J, Kirkpatrick D, Olszens K, Shao H, Pao YH, and Nadeau JH. Naturally occurring variation in cardiovascular traits among inbred mouse strains. Genomics 79: 679-685, 2002.

37. Itokawa K, Sora I, Schindler CW, Itokawa M, Takahashi N, and Uhl GR. Heterozygous VMAT2 knockout mice display prolonged QT intervals: possible contributions to sudden death. Mol Brain Res 71: 354-357, 1999.

38. James JF, Hewett TE, and Robbins J. Cardiac physiology in transgenic mice. Circ Res 82: 407-415, 1998.

39. Johansson C, Gothe S, Forrest D, Vennstrom B, and Thoren P. Cardiovascular phenotype and temperature control in mice lacking thyroid hormone receptor- $\beta$ or both $\alpha 1$ and $\beta$. Am J Physiol Heart Circ Physiol 276: H2006-H2012, 1999.

40. Jumrussirikul P, Dinerman J, Dawson TM, Dawson VL, Ekelund U, Georgakopoulos D, Schramm LP, Calkins H, Snyder SH, Hare JM, and Berger RD. Interaction between neuronal nitric oxide synthase and inhibitory $\mathrm{G}$ protein activity in heart rate regulation in conscious mice. J Clin Invest 102: 1279-1285, 1998.

41. Just A, Faulhaber J, and Ehmke H. Autonomic cardiovascular control in conscious mice. Am J Physiol Regul Integr Comp Physiol 279: R2214-R2221, 2000.

42. Kant GJ, Pastel RH, Bauman RA, Meininger GR, Maughan KR, Robinson TN III, Wright WL, and Covington PS. Effect of chronic stress on sleep in rats. Physiol Behav 57: 359-365, 1995.

43. Kinter LB. Cardiovascular telemetry and laboratory animals welfare: New reduction and refinement alternatives (Abstract). In: General Pharmacology/Safety Pharmacology Meeting. Philadelphia, PA: 1996.

44. Kinter LB, Murphy DJ, Mann WA, Leonard TB, and Morgan DG. Major organ system toxicology: an integrated approach to pharmacodynamic safety assessment in animals. In: Toxicological Testing and Evaluation. New York: Elsevier, 1997, p. $155-168$

45. Kinter LB and Johnsen DK. Remote monitoring of experimental endpoints in animals using radio-telemetry and bioimped- ance technology. In: Humane Endpoints in Animal Experiments for Biomedical Research. London: RSM, 1999, p. 58-65.

46. Kinter LB and Valentin JP. Safety pharmacology and risk assessment. Fundam Clin Pharmacol 16: 175-182, 2002.

47. Kramer K, Van Acker SABE, Voss HP, Grimbergen JA, Van der Vijgh WJF, and Bast A. Use of telemetry to record electrocardiogram and heart rate in freely moving mice. J Pharmacol Toxicol Methods 30: 209-215, 1993.

48. Kramer K, Grimbergen JA, Brockway RV, Brockway BP, and Voss HP. Circadian respiratory rate rhythms in freely moving small laboratory animals using radio-telemetry. Lab Anim 28: 38-41, 1999

49. Kramer K. Applications and Evaluation of Radio-Telemetry in Small Laboratory Animals (PhD thesis). Utrecht, The Netherlands: University of Utrecht, 2000.

50. Kramer K, Voss P, Grimbergen JA, Huetteman DA, Mills PA, Zwiers LM, and Brockway BP. Telemetric monitoring of blood pressure in freely moving mice: a preliminary study. $L a b$ Anim 34: 272-280, 2000.

51. Kramer K, Kinter L, Brockway BP, Voss HP, Remie R, and Van Zutphen LFM. The use of radiotelemetry in small laboratory animals: recent advances. Contemp Top Lab Anim Sci 40: 8-16, 2001.

52. Krege JH, Hodgin JB, Hagaman JR, and Smithies O. A noninvasive computerized tail-cuff system for measuring blood pressure in mice. Hypertension 25: 1111-1115, 1995.

53. Kvetnansky R, Sun CL, Torda T, and Kopin IJ. Plasma epinephrine and noradrenaline levels in stressed rats- effect of adrenalectomy. Pharmacologist 19: 241-245, 1977.

54. Lamprecht F, Williams RB, and Kopin IJ. Serum dopamine$\beta$-hydroxylase during development of immobilization-induced hypertension. Endocrinology 92: 953-956, 1973.

55. Langenbach GEJ, Van Ruijven LJ, and Van Eijden TMGJ. A telemetry system to chronically record muscle activity in middle-sized animals. J Neurosci Methods 114: 197-203, 2002.

56. Lawson DM, Churchill M, and Churchill PC. The effects of housing enrichment on cardiovascular parameters in spontaneously hypertensive rats. Contemp Top Lab Anim Sci 39: 9-13, 2000.

57. Lemmer B, Mattes A, Bohm M, and Gante D. Circadian blood pressure variation in transgenic hypertensive rats. Hypertension 22: 97-101, 1993.

58. Lemmer $\mathbf{B}$ and Witte $\mathbf{K}$. Telemetric data acquisition of cardiovascular function in biology and pharmacology. In: Animal Alternatives, Welfare and Ethics. Amsterdam: Elsevier, 1997, p. 311-320.

59. Livezey GT and Sparber SB. Hyperthermia sensitizes rats to cocaine's proconvulsive effects and unmasks EEG evidence of kindling after chronic cocaine. Pharmacol Biochem Behav 37: 761-767, 1990.

60. Mansier P, Medigue C, Charlotte N, Vermeiren C, Coaboeuf E, Deroubai E, Ratner E, Chevalier B, Clairambault J, Carre F, Dahkli T, Bertin B, Briand P, Strosberg D, and Swynghedauw B. Decreased heart rate variability in transgenic mice overexpressing arterial $\beta 1$-adrenoceptors. Am $J$ Physiol Heart Circ Physiol 271: H1465-H1572, 1996.

61. Meile T and Zittel TT. Telemetric small intestinal motility recording in awake rats. Eur Surg Res 34: 271- 274, 2002.

62. Mills PA, Huetteman DA, Brockway BP, Zwiers LM, Gelsema AJM, Schwartz RS, and Kramer K. A new method for measurement of blood pressure, heart rate, and activity in the mouse by radio-telemetry. J Appl Physiol 88: 1537-1544, 2000.

63. Mitchell GF, Jeron A, and Koren G. Measurement of heart rate and Q-T interval in the conscious mouse. Am J Physiol Heart Circ Physiol 274: H747-H751, 1998.

64. Moran MM, Roy PR, Wade CE, Corbin BJ, and Grineland RE. Size constraints of telemeters in rats. J Appl Physiol 85: 1564-1571, 1998.

65. Morgan DG, Kelvin AS, Kinter LB, Fish CJ, Kems WD, and Rhodes G. The application of toxicokinetic data to dosage selection in toxicology studies. Toxicol Pathol 22: 112-123, 1994. 
66. Mumford $\mathbf{H}$ and Wetherell JR. A simple method for measuring EEG in freely moving guinea pigs. J Neurosci Methods 107: $125-130,2001$.

67. Murphy DJ, Renninger JP, and Gossett KA. A novel method for chronic measurement of pleural pressure in conscious rats. J Pharmacol Toxicol Methods 39: 137-141, 1998.

68. Nakajima M, Sakai T, Mizumoto A, and Itoh Z. Development of a new telemetry recording system for measuring of gastrointestinal contractile activity in unrestrained and conscious small animals. J Smooth Muscle Res 32: 1-7, 1996.

69. Nguyên-Trân VTB, Kubalak SW, Minamisawa S, Fiset C, Wollert KC, Brown AB, Ruiz-Lozano P, Barrere-Lemaire S, Kondo R, Norman LW, Gourdie RG, Rahme MM, Feld GK, Clark RB, Giles WR, and Chien KR. A novel genetic pathway for sudden cardiac death via defects in the transition between ventricular and conduction system cell lineages. Cell 102: 671-682, 2000.

70. O'Neil PJ and Kaufman LN. Effects of indwelling arterial catheters or physical restraint on food consumption and growth patterns of rats: Advantages of noninvasive blood pressure measurements techniques. Lab Anim Sci 40: 641-643, 1990.

71. Rowan A. The concepts of animal welfare and animal suffering. In: Animal Alternatives, Welfare and Ethics. Amsterdam: Elsevier, 1997, p. 157-168.

72. Russell WMS and Burch RL. The Principles of Humane Experimental Technique. London: Methuen, 1959.

73. Saba S, Wang PJ, and Estes NA III. Invasive cardiac electrophysiology in the mouse: techniques and applications. Trends Cardiovasc Med 10: 122-132, 2000.

74. Schlingmann F, Van de Weerd HA, Baumans V, Remie R, and Van Zutphen LFM. A balance device for the analysis of behavioural patterns of the mouse. Anim Welfare 7: 177-188, 1998.

75. Schnell CR and Gerber P. Training and remote monitoring of cardiovascular parameters in non-human primates. Prim Rep 49: 61-70, 1997.

76. Sei $\mathbf{H}$ and Morita Y. Acceleration of EEG theta wave precedes the phasic surge of arterial pressure during REM sleep in the rat. Neuroreport 7: 3059-3062, 1996.

77. Sheldon JH, Lapp RW, Perrone MH, and Cox BF. Effects of tethering on cardiovascular responses to antihypertensive agents in the conscious telemetry-instrumented spontaneously hypertensive rat (SHR) (Abstract). FASEB J 15: 852, 1991.

78. Shults MC, Rhodes RK, Uptike SJ, Gilligan BJ, and Reining WN. A telemetry-instrumentation system for monitoring multiple subcutaneously implanted glucose sensors. IEEE Trans Biomed Eng 41: 937-942, 1994.

79. Shusterman V, Usiene I, Harrigal C, Lee JS, Kubota T, Feldman AM, and London B. Strain-specific patterns of autonomic nervous system activity and heart failure susceptibility in mice. Am J Physiol Heart Circ Physiol 282: H2076-H2083, 2002. First published February 14, 2002; 10.1152/ajpheart. 00917.2001.

81. Stauss HM, Godecke A, Mrowka R, Schrader J, and Persson PB. Enhanced blood pressure variability in eNOS knockout mice. Hypertension 33: 1359-1363, 1999.

82. Taché Y, Du Ruisseau P, Ducharme JR, and Collu R. Pattern of adenohypophyseal hormone changes in male rats following chronic stress. Neuroendocrinology 26: 208-219, 1978.

83. Timofeeva OA and Gordon CJ. Changes in EEG power spectra and behavioural states in rats exposed to the acethylcholinesterase inhibitor chlorpyrifos and muscatinic agonist oxotremorine. Brain Res 893: 165-177, 2001.

84. Uechi M, Asai K, Osaka M, Smith A, Sato N, Wagner TE, Ishikawa Y, Hayakawa H, Vatner DE, Shannon RP, Homcy CJ, and Vatner SF. Depressed heart rate variability and arterial baroflex in conscious transgenic mice with overexpression of cardiac $\left(\mathrm{G}_{\mathrm{s} \alpha}\right)$. Circ Res 82: 416-423, 1998.

85. Van Acker SABE, Kramer K, Grimbergen JA, Zhang J, Van der Vijgh WJF, and Bast A. Doxorubicin-induced cardiotoxicity monitored by ECG in freely moving mice. A new model to test potential protectors. Cancer Chemother Pharmacol 38: 95$101,1996$.

86. Van Dongen JJ, Remie R, Rensema JW, and Wunnik GHJ. Manual of Microsurgery on the Laboratory Rat. Amsterdam: Elsevier, 1990.

87. Van de Weerd HA. Environmental Enrichment for Laboratory Mice: Preferences and Consequences (PhD thesis). Utrecht, The Netherlands: University of Utrecht, 1996.

88. Ward HB, Baker TG, and McLaren A. A histological study of the gonads of T16H/XSxr hermaphrodite mice. J Anat 158: $65-75,1988$

89. Wickman K, Nemec J, Gendler SJ, and Clapham DE. Abnormal heart rate regulation in GIRK4 knockout mice. Neuron 20: 103-114, 1998.

90. Weigler BJ. Justifying the number of animals in IACUC proposals. Contemp Top Lab Anim Sci 34: 47-50, 1995.

91. Witte $\mathbf{K}$ and Lemmer B. Free-running rhythms in blood pressure and heart rate in normotensive and transgenic hypertensive rats. Chronobiol Int 12: 237-247, 1995. 